\title{
Tuning Charge Transport in Solution-Sheared Organic Semiconductors Using Lattice Strain
}

\section{Citation}

Giri, Gaurav, Eric Verploegen, Stefan C. B. Mannsfeld, Sule Atahan-Evrenk, Do Hwan Kim, Sang Yoon Lee, Hector A. Becerril, Alán Aspuru-Guzik, Michael F. Toney, and Zhenan Bao. 2011.

Tuning charge transport in solution-sheared organic semiconductors using lattice strain. Nature 480(7378): 504-508.

\section{Published Version}

doi:10.1038/nature10683

\section{Permanent link}

http://nrs.harvard.edu/urn-3:HUL.InstRepos:8365120

\section{Terms of Use}

This article was downloaded from Harvard University's DASH repository, and is made available under the terms and conditions applicable to Open Access Policy Articles, as set forth at http:// nrs.harvard.edu/urn-3:HUL.InstRepos:dash.current.terms-of-use\#OAP

\section{Share Your Story}

The Harvard community has made this article openly available.

Please share how this access benefits you. Submit a story.

\section{Accessibility}




\title{
Strained Molecular Packing of TIPSE-pentacene with Enhanced Charge Transport Using Solution Shearing
}

Gaurav Giri $^{1}$, Eric Verploegen ${ }^{1,2}$, Stefan Mannsfeld ${ }^{2}$, Hector A. Becerril ${ }^{3}$, Sule Atahan-Evrenk ${ }^{7}$, Jonathan Rivnay ${ }^{4}$, Do Hwan $\mathrm{Kim}^{1}$, Sang Yoon Lee ${ }^{5}$, Alberto Salleo ${ }^{4}$, John E. Anthony ${ }^{6}$, Alan Aspuru-Guzik ${ }^{7}$, Michael F. Toney ${ }^{2}$, Zhenan Bao ${ }^{1}$

${ }^{1}$ Department of Chemical Engineering, Stanford University, Stanford, CA 94305, ${ }^{2}$ SLAC National Accelerator Laboratory, Palo Alto, CA 94304, ${ }^{3}$ Department of Chemistry, Brigham Young University - Idaho, Rexburg, ID 83460, ${ }^{4}$ Department of Materials Science and Engineering, Stanford University, Stanford, CA 94305, ${ }^{5}$ Samsung Advanced Institute of Technology, Mt. 14-1, Nongseo-Ri, Giheung-Eup, Yongin-Si, Kyunggi-Do 449-712, South Korea, ${ }^{6}$ Department of Chemistry, University of Kentucky, Lexington, KY 40506, ${ }^{7}$ Department of Chemistry and Chemical Biology, Harvard University, Cambridge, MA 02138

\begin{abstract}
For organic semiconductors (OSCs), the molecular packing determines the charge transport of the resulting devices. It is desirable to control the molecular packing of small molecular OSCs through facile processing methods in order to tune the electrical properties of OSC devices. We describe the alteration of the 5,12-bis(triisopropylsilylethynyl) pentacene (TIPSE-pentacene) molecular packing by changing the conditions used during solution shearing deposition. Solution shearing deposits TIPSE-pentacene thin film in a non-equilibrium state, and the $\pi$ - $\pi$ stacking distance between the molecules can be tuned between $3.08 \AA$ to $3.33 \AA$ as a function of these conditions, which in turn significantly affects the electronic properties of TIPSE-pentacene. Using the shearing speed as a handle for manipulating the molecular packing,
\end{abstract}


the charge carrier mobility was increased from $0.3 \mathrm{~cm}^{2} / \mathrm{Vs}$ to a record high mobility for TIPSEpentacene at $4.6 \mathrm{~cm}^{2} / \mathrm{Vs}$. Control of the molecular packing using processing conditions will allow for the development of high performance OSC devices beyond traditional synthetic methods. 
Inorganic semiconductor films that exhibit lattice strain can show improved charge carrier mobility compared to the same semiconductor exhibiting an unstrained lattice.(1-2) Inorganic semiconductors are often strained due to lattice mismatch that occurs when the semiconductor crystal is grown by heteroepitaxy. Recently, the bandgap of graphene has been modified using uniaxial strain.(3) Such strain effects may be possible for OSCs. However, it has not been reported. Straining the molecular packing of OSCs can alter the $\pi-\pi$ stacking distance, and the change in this distance and the relative positions of adjacent molecules can significantly affect the charge transfer integral of OSCs (4-7). The charge transfer integral, which describes the electronic wavefunction overlap between adjacent molecules, is crucial in determining charge carrier mobility.

Changing the chemical structure of the OSC as well as the chemical structure of the dielectric interface can affect the molecular packing of OSCs. They are effective methods used to design high performance OSCs.(6, 8-12) Non-synthetic techniques offer an alternative for controlling the molecular packing. In biological systems the molecular packing of protein crystals can be altered by tuning the crystal growth conditions, such as $\mathrm{pH}$, solvent, concentration, and temperature, yielding lattice parameter shifts of up to $10 \%$.(13) Similarly, some OSCs exhibit solid-state transitions where discrete molecular packing motifs are stable at specific temperatures, and also, the thin film packing may be different from the bulk molecular packing motif.(14-16) Here, we report the formation of strained molecular packing of TIPS-pentacene by using a solution shearing method.(17) The $\pi-\pi$ stacking distance decreased from $3.33 \AA$ for evaporated thin film on $\mathrm{SiO}_{2}$ to $3.08 \AA$ for the solution sheared thin film prepared at a shearing speed of $8 \mathrm{~mm} / \mathrm{s} .(18)$ The charge carrier mobility was increased from $0.3 \mathrm{~cm}^{2} / \mathrm{Vs}$ for unstrained films to as high as $4.6 \mathrm{~cm}^{2} / \mathrm{Vs}$ for strained, aligned thin films. 
During the solution shearing process a shearing plate drags the solution across a heated substrate while keeping the bulk of the solution between the plate and the substrate, with only the evaporation front exposed (Fig. S1, Fig. 1A).(17, 19) We found that a variety of process parameters can be systematically changed to influence thin film crystallite texture and molecular packing. The parameters include substrate temperature, shearing speed, solvent, solution concentration, gap distance between the plate and the substrate, and the tilt angle between the plate and the substrate. Here, we report on using shearing speed as the main parameter, as changing the shearing speed incrementally changes the molecular packing as well as the crystal texture of the TIPSE-pentacene thin films.

The solution sheared films were characterized using a cross polarized optical microscope (CPOM) to determine the size and orientation of the crystallites. Fig. 1, B-F shows CPOM images illustrating the crystallite texture of thin films prepared as a function of shearing speed, ranging from $0.4 \mathrm{~mm} / \mathrm{s}$ to $8 \mathrm{~mm} / \mathrm{s}$. Low magnification scans are shown in Fig. S2. At a shearing speed of $0.4 \mathrm{~mm} / \mathrm{s}$ (Fig. 1B), the TIPSE-pentacene crystallites are up to a millimeter wide and up to a centimeter long, with the long axis in the shearing direction. Sample rotation under cross polarized light indicates that the TIPSE-pentacene crystallites are well oriented relative to each other and along the shearing direction.(20) Shearing at a speed of $1.6 \mathrm{~mm} / \mathrm{s}$ (Fig. 1C) results in crystallites narrower and shorter in length than crystallites resulting from a shearing speed of 0.4 $\mathrm{mm} / \mathrm{s}$. This trend in crystallite texture continues up to a shearing speed of $2.8 \mathrm{~mm} / \mathrm{s}$ (Fig. 1D).

When the shearing speed reaches $4 \mathrm{~mm} / \mathrm{s}$, a comet shaped morphology (known as 'transcrystalline') is observed (Fig. 1E). These features are hundreds of microns wide and several millimeters long. Such transcrystalline textures have been observed for polymers crystallized in a temperature gradient (21-22) and for small molecules crystallized from a 
supersaturated solution which forms in the presence of a concentration gradient.(23) A similar concentration gradient between the bulk solution and the evaporation front forms during solution shearing.(23-24) Further increase in the shearing speed to $8 \mathrm{~mm} / \mathrm{s}$ results in an isotropic, spherulitic crystallite texture (Fig. 1F); no preferential orientation relative to the shearing direction is observed for the spherulites.

In addition to altering the TIPSE-pentacene film crystallite texture, the solution shearing speed also has an effect on the molecular packing in the film. X-ray scattering experiments were performed to characterize the crystallite orientation and molecular packing. The molecular packing structure of the TIPS-pentacene unit cell was previously calculated for the evaporated thin film.(18) Here, the same technique was applied to the sheared films. The evaporated TIPSEpentacene films (Fig. 2A) exhibit a slipped-stack packing motif similar to that of the TIPSEpentacene bulk. Solution shearing results in a more oblique molecular packing motif, accompanied by a change in the $\pi-\pi$ stacking distance between the TIPSE-pentacene molecules (Fig. 2B). The smallest $\pi-\pi$ stacking distance decreases from $3.33 \AA$ in the evaporated thin film to $3.08 \AA$ in the thin film prepared at a shearing speed of $8 \mathrm{~mm} / \mathrm{s}$. This results in an increase in the HOMO orbital overlap as seen from the increased charge transfer integral, resulting in a faster charge transfer between two cofacially stacked molecules. The charge transfer integral changes from $11.7 \mathrm{meV}$ in the evaporated thin film to $36.9 \mathrm{meV}$ in the sheared thin film (Fig. S3, Table S1).

A least-square-error fitting procedure was used to determine the unit cell of the solution sheared TIPS-pentacene thin films from grazing incidence X-ray diffraction (GIXD) data. As the shearing speed increases, the (100) d-spacing shifts incrementally to lower values, from $7.69 \AA$ to $7.25 \AA$, while concurrently, the (010) d-spacing shifts incrementally to higher values, from 
$7.83 \AA$ to $8.56 \AA$ (Fig. 3A, Table S2). These d-spacing shifts occur in plane only, as the vertical layer spacing, also known as the (001) d-spacing, does not systematically shift with the shearing speed (Fig. S4).(19) The in-plane d-spacing shifts indicate that the TIPSE-pentacene in-plane unit cell geometry undergoes an monotonically increasing strain, becoming more oblique with increasing shearing speed, while the in-plane unit cell area remains comparatively constant (Table 1).

We show that the change in the position of GIXD signal used to determine the d-spacing shifts is not due to the existence of two crystal polymorphs whose individual contribution changes at different shearing speeds (Fig. S5). Films of different thickness can exhibit different levels of crystal polymorphs that can affect the GIXD signal, so this effect is also considered. We find that the film thickness does not play a role in the change in molecular packing, as the majority of the molecular packing shift occurs at roughly the same thin film thickness (Fig. S6). We also exclude the possibility of an interface layer of TIPSE-pentacene at the dielectric/semiconductor interface that may have a different molecular packing compared to the bulk crystal. The interface layer would not have significantly changed GIXD pattern. The possibility of solvent cocrystallization that changes the molecular packing must also be considered, but the unit cell calculations do not show in-plane area expansion, which is consistent showed an excellent fit without the inclusion of solvent molecules. Moreover, the film sheared at a low speed $(0.4 \mathrm{~mm} / \mathrm{s})$ showed the same GIXD pattern as that of evaporated thin film, which had no solvent inclusion.

After confirming the strained molecular packing structure of the TIPSE-pentacene thin films, we measured the charge transport properties of these films. The in-plane charge carrier mobility was measured using bottom gate, top contact field effect transistors.(19) The average mobility as a function of shearing speed was found to increase until reaching a maximum value of $2.1 \mathrm{~cm}^{2} / \mathrm{Vs}$ 
for thin films prepared at a speed of $2.6 \mathrm{~mm} / \mathrm{s}$ (Fig. 3A, Table S3). The mobility decreased for faster shearing speeds, reaching an average value of $0.47 \mathrm{~cm}^{2} / \mathrm{Vs}$ for thin films prepared at a speed of $8 \mathrm{~mm} / \mathrm{s}$. The best performing device showed a mobility of $4.6 \mathrm{~cm}^{2} / \mathrm{Vs}$, deposited at a shearing speed of $2.8 \mathrm{~mm} / \mathrm{s}$ (Fig. 3B). The decrease in the average mobility observed at shearing speeds above $2.6 \mathrm{~mm} / \mathrm{s}$ is attributed to the decrease in crystallite alignment and size, as well as the increase in the number of grain boundaries (Fig. 1).(20)

To determine if the strained molecular packing is a result of a TIPSE-pentacene molecules present in a metastable state, we exposed the films to toluene vapor, which induced a reorganization of the molecular packing without affecting the crystallite texture. GIXD images were taken of thin films before, during, and after exposure of the film to toluene vapor. Upon exposure to toluene vapor a rearrangement takes place, resulting in a molecular packing structure similar to that of the evaporated film (Video S1).(19) The molecular packing for the strained thin films returned to a similar GIXD pattern and packing structure as the evaporated film after exposure to toluene for an hour (Fig. S7).

The mobility of the TIPS-pentacene TFTs was also measured before and after exposure to toluene vapor. A decrease in TFT mobility was observed for thin films prepared at a speed above $0.4 \mathrm{~mm} / \mathrm{s}$ (Fig. S8). The reduction in mobility after toluene vapor exposure is consistent with the observation of reduced molecular packing distortion. CPOM and atomic force microscopy were used to confirm that no cracks were formed in the film, so that the change in mobility can be attributed to the reduction in molecular packing strain (Fig. S9). The mobility was measured after 5 min to exposure to toluene, as longer exposure times formed cracks in the thin film. The thin films prepared at a shearing speed of $0.4 \mathrm{~mm} / \mathrm{s}$ show a slight increase in mobility after toluene vapor exposure, which is attributed to previously reported improvement of 
crystallinity.(25) This result indicates that the alteration of molecular packing induced by solution shearing speeds above $0.4 \mathrm{~mm} / \mathrm{s}$ is the key factor that causes increased charge carrier mobility, until the mobility starts to decrease due to grain boundaries.

In our solution shearing method, the liquid film thickness decreases as a function of increasing shearing speed.(23) A steeper temperature gradient is established in the thinner film, as the bottom of the film is in contact with a heating source, while the top surface is exposed to ambient temperature. The solvent evaporates faster in the thinner film, which results in a faster solvent and OSC flow towards the growing crystal front. (Fig. S1) The faster solvent evaporation rate also gives the growing crystal front less time to reach an equilibrium state. Consequently, the growing crystal front crystallizes in a metastable state. Faster rates of solvent evaporation coupled with velocity and concentration gradients gave rise to strained and aligned crystals.

The direction of elongated crystallite texture growth is perpendicular to the (100) d-spacing, which is also the direction of high charge transport. $(18,26)$ As shearing speed increases, this dspacing as well as the $\pi-\pi$ stacking distance between the TIPSE-pentacene molecules decreases. Consequently, the charge transfer integral increases, resulting in higher charge carrier mobilities.(4) Here, we have demonstrated that by varying simple processing conditions, we can incrementally change the molecular packing and the $\pi-\pi$ stacking distance of the 2-D brick wall packing TIPSE-pentacene. The $\pi$ - $\pi$ stacking distance decreased from $3.35 \AA$ to $3.08 \AA$. We were able to achieve an average charge carrier mobility of $2 \mathrm{~cm}^{2} / \mathrm{Vs}$. The highest value we achieved is $4.6 \mathrm{~cm}^{2} / \mathrm{Vs}$ compared to $1.8 \mathrm{~cm}^{2} / \mathrm{Vs}(27)$ as the best reported in literature. However, we did not observe a monotonic increase in mobility as the shearing speed increased because the crystallite size decreased and they became less oriented. The increased number of grain boundaries hindered charge transport within the TFT. The effect of grain boundaries on charge carrier 
mobilities has been well established elsewhere (28-29). Our solution shearing deposition is a general method that can be potentially applied to a variety of systems to systematically tune molecular packing. The solution shearing method is a facile method for exploring new parameters in solution processing of OSCs. The fact that the solution is sandwiched between two substrates allows a broader range of temperature and shearing speeds to be sampled compared to other commonly used solution processing methods, such as dip coating and spin-coating. Changing molecular packing without changing the chemical structure provides a method of understanding charge transport and improvement of the electrical characteristics of OSCs for practical applications. 

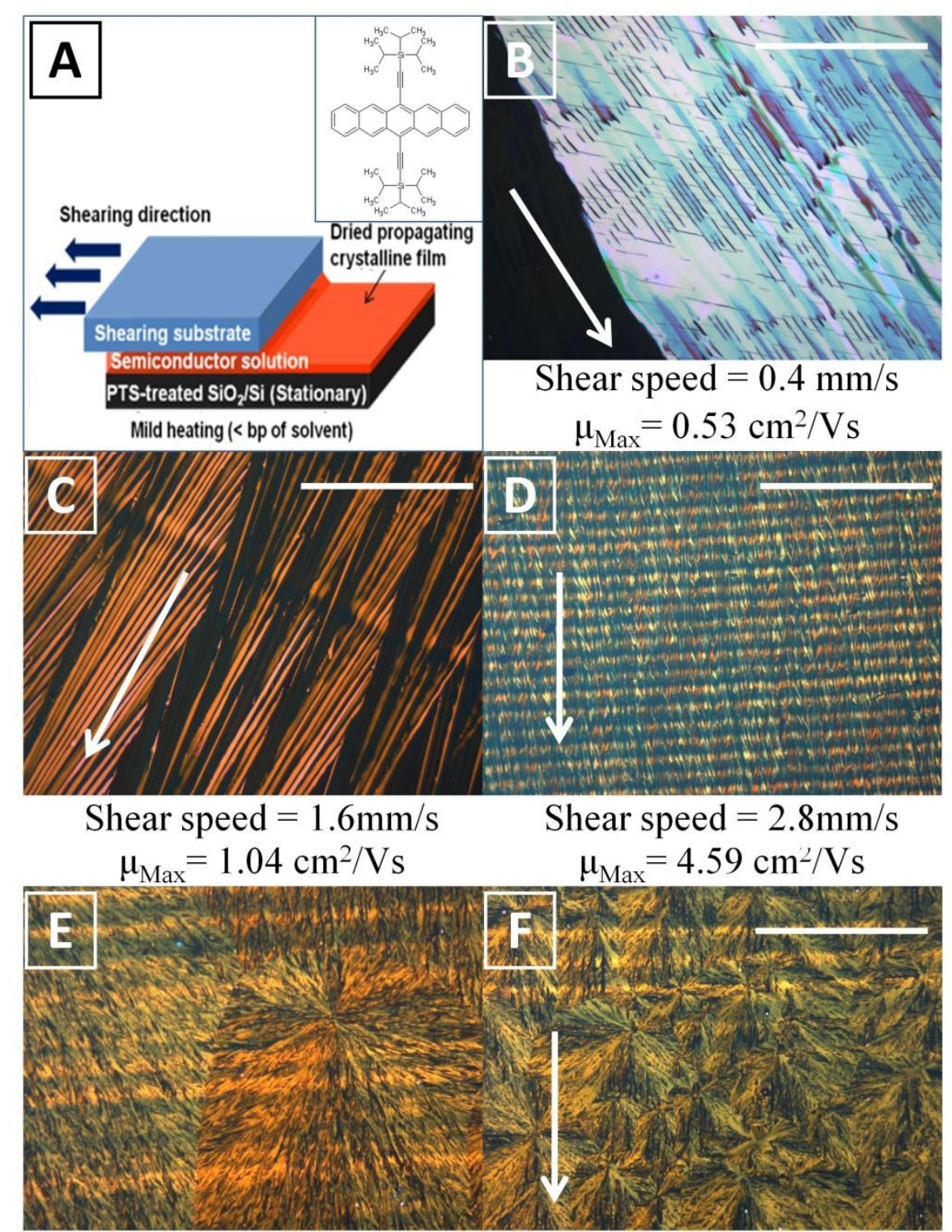

$$
\begin{array}{cc}
\text { Shear speed }=4 \mathrm{~mm} / \mathrm{s} & \text { Shear speed }=8 \mathrm{~mm} / \mathrm{s} \\
\mu_{\mathrm{Max}}=2.78 \mathrm{~cm}^{2} / \mathrm{Vs} & \mu_{\mathrm{Max}}=1.21 \mathrm{~cm}^{2} / \mathrm{Vs}
\end{array}
$$

Fig. 1: (A) Schematic diagram of solution shearing method. (B) - (F) Cross polarized optical micrographs of solution sheared TIPSE-pentacene thin films, formed with shearing speed $0.4 \mathrm{~mm} / \mathrm{s}, 1.6 \mathrm{~mm} / \mathrm{s}, 2.8 \mathrm{~mm} / \mathrm{s}, 4 \mathrm{~mm} / \mathrm{s}$ and $8 \mathrm{~mm} / \mathrm{s}$, respectively (scale bar is $200 \mu \mathrm{m}$ ). Dark regions of the images are due to crystallites oriented along the polarization direction of the light. In all cases the white arrow represents the shearing direction. 

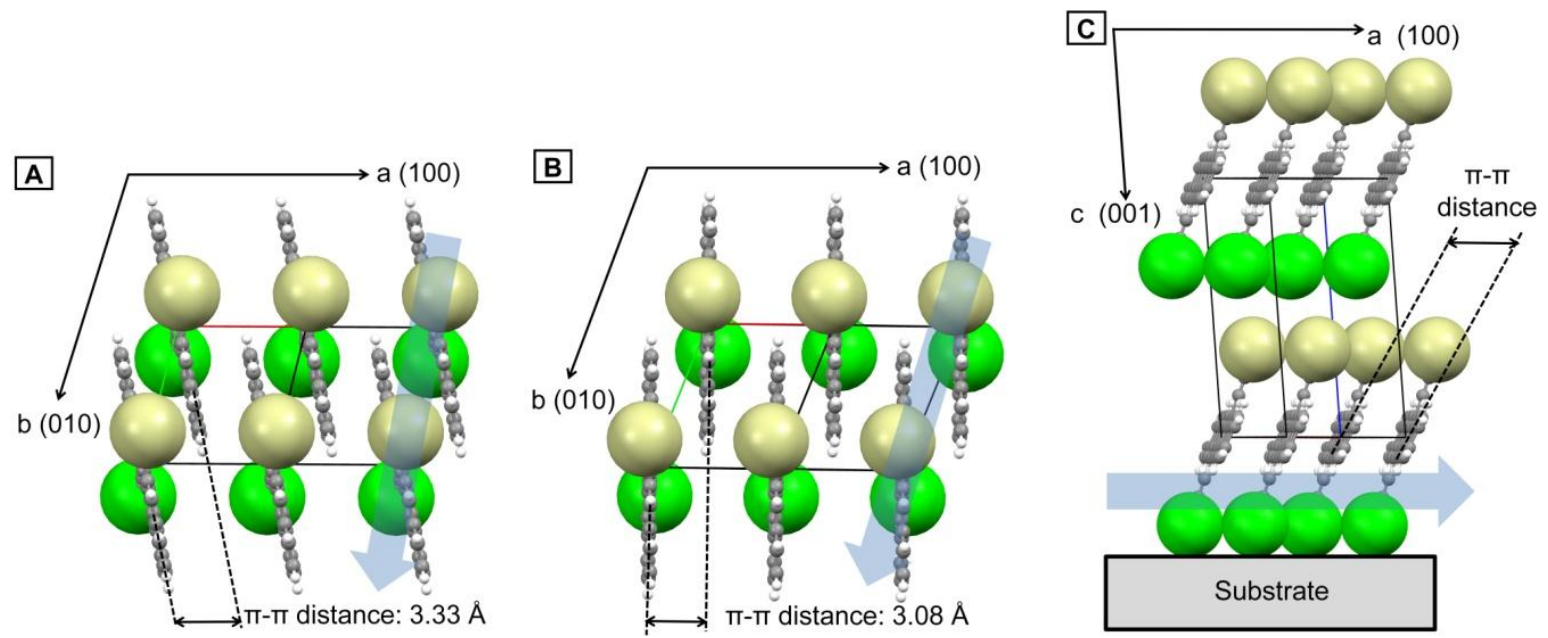

Fig. 2: Molecular packing structure of TIPSE-pentacene prepared under different conditions. (A) Evaporated thin film. (B) Solution sheared film at $8 \mathrm{~mm} / \mathrm{s}$. (C) Solution sheared film at $8 \mathrm{~mm} / \mathrm{s}$, viewed along the $\mathrm{b}$ axis. The sphere represents the TIPSE group. The yellow and green colors correspond to the front and back of the molecule, respectively. The blue arrow in (c) represents the high charge transport direction, which is also the direction of shearing. 

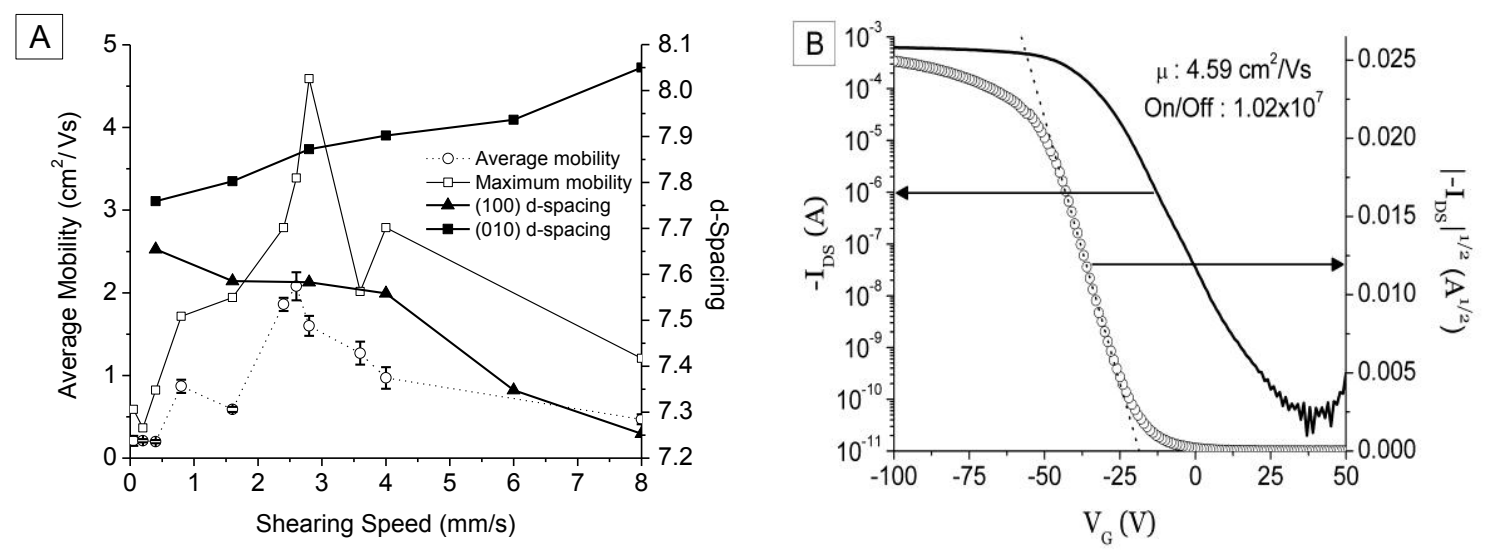

Fig. 3: (A) Charge carrier mobilities (left axis) and d-spacing (right axis) of TIPSE-pentacene thin films sheared at different speeds. The error bars show the standard error of the mean. (B) Transfer curve of a device prepared at $2.8 \mathrm{~mm} / \mathrm{s}$ shearing speed showing mobility of 4.59 $\mathrm{cm}^{2} / \mathrm{Vs}$. 


\begin{tabular}{|c|c|c|c|c|c|c|c|c|}
\hline $\begin{array}{c}\text { Speed } \\
(\mathbf{m m} / \mathbf{s})\end{array}$ & $\begin{array}{c}\mathbf{a} \\
(\mathbf{\AA})\end{array}$ & $\begin{array}{c}\mathbf{b} \\
(\mathbf{A})\end{array}$ & $\begin{array}{c}\mathbf{c} \\
(\mathbf{\AA})\end{array}$ & $\begin{array}{c}\boldsymbol{\gamma} \\
(\mathbf{d e g})\end{array}$ & $\begin{array}{c}\text { In-plane area } \\
\left(\mathbf{\AA}^{2}\right)\end{array}$ & $\begin{array}{c}\text { Mobility } \\
\left(\mathbf{c m}^{2} / \mathbf{V s}\right)\end{array}$ & $\begin{array}{c}\text { On/Off } \\
\mathbf{V}\end{array}$ & $\begin{array}{c}\mathbf{V t} \\
(\mathbf{V})\end{array}$ \\
\hline Evaporated(18) & 7.70 & 7.83 & 16.78 & 99.0 & 59.5 & - & - & - \\
\hline Single Crystal(18) & 7.75 & 7.56 & 17.01 & 96.4 & 58.2 & - & - & - \\
\hline 0.4 & 7.73 & 7.79 & & 97.8 & 59.7 & 0.335 & $1.8 \mathrm{E}+04$ & 39.3 \\
\hline 1.6 & 7.68 & 7.97 & & 99.8 & 60.3 & 0.778 & $2.1 \mathrm{E}+05$ & 30.2 \\
\hline 2.8 & 7.56 & 8.04 & & 100.2 & 59.9 & 1.490 & $8.2 \mathrm{E}+06$ & -16.5 \\
\hline 4 & 7.59 & 8.16 & & 100.7 & 60.9 & 0.813 & $1.0 \mathrm{E}+07$ & -27.4 \\
\hline 8 & 7.56 & 8.56 & 16.83 & 108.0 & 61.5 & 0.471 & $1.2 \mathrm{E}+07$ & -35.7 \\
\hline $\begin{array}{c}8-T o l u e n e ~ V a p o r \\
\text { Annealed 1hr }\end{array}$ & 7.80 & 7.82 & 17.00 & 98.7 & 60.3 & - & - & - \\
\hline
\end{tabular}

Table 1: TIPSE-pentacene unit cell parameters and the corresponding TFT performance (average mobility, on/off ratio and threshold voltage) as a function of different shearing speeds. 


\section{Acknowledgements:}

We thank M. Michele Nelson in 3M Co. for providing high purity TIPSE-pentacene. This publication was partially supported by the National Science Foundation DMR-Solid State Chemistry (DMR-0705687-002), the Samsung Advanced Institute of Technology, , the Global Climate and Energy Project at Stanford University (SPO 25591130-45282-A), the Air Force Office of Scientific Research (Award no. FA9550-09-1-0256) and the Center for Advanced Molecular Photovoltaics (Award no. KUSC1-015-21) sponsored by King Abdullah University of Science and Technology (KAUST). EV would like to thank the Eastman Kodak Corporation and the Kodak Fellows Program for support. ZB acknowledges support from the David Filo and Jerry Yang Faculty Fellowship from Stanford University. Portions of this research were carried out at the Stanford Synchrotron Radiation Lightsource, a national user facility operated by Stanford University on behalf of the U.S. Department of Energy, Office of Basic Energy Sciences.

Any opinions, findings, and conclusions or recommendations expressed in this publication are those of the author(s) and do not necessarily reflect the views of Stanford University, the Sponsors of the Global Climate and Energy Project, or others involved with the Global Climate and Energy Project.

1. A. A. A. S. Zoolfakar, paper presented at the 2009 5th International Colloquium on Signal Processing \& Its Applications, Kuala Lampur, Malaysia, 20092009.

2. M. L. Lee, E. A. Fitzgerald, M. T. Bulsara, M. T. Currie, A. Lochtefeld, Journal of Applied Physics 97, 011101 (2005).

3. Z. H. Ni et al., ACS Nano 2, 2301 (2008).

4. J. L. Bredas, J. P. Calbert, D. A. da Silva Filho, J. Cornil, Proc Natl Acad Sci U S A 99, 5804 (Apr 30, 2002).

5. D. Andrienko, J. Kirkpatrick, V. Marcon, J. Nelson, K. Kremer, physica status solidi (b) 245, 830 (2008).

6. $\quad$ C. Reese, M. E. Roberts, S. R. Parkin, Z. Bao, Advanced Materials 21, 3678 (2009).

7. R. A. Marcus, The Journal of Chemical Physics 24, 966 (1956).

8. J. E. Anthony, D. L. Eaton, S. R. Parkin, Org Lett 4, 15 (Jan 10, 2002).

9. M. L. Tang, J. H. Oh, A. D. Reichardt, Z. Bao, Journal of the American Chemical Society 131, 3733 (2009).

10. T. Okamoto et al., Advanced Materials 19, 3381 (2007).

11. X. Feng et al., Nat Mater 8, 421 (2009).

12. M. Gsänger et al., Angewandte Chemie 122, 752 (2010).

13. C. C. I. Dobrianov, S.G. Lemay, K.D. Finkelstein, R.E. Thorne, Journal of Crystal Growth 196, 13 (1999).

14. Q. Yuan et al., J Am Chem Soc 130, 3502 (Mar 19, 2008).

15. S. C. B. Mannsfeld, A. Virkar, C. Reese, M. F. Toney, Z. Bao, Advanced Materials 21, 2294 (2009).

16. C. R. Newman et al., Chemistry of Materials 16, 4436 (2004).

17. H. A. Becerril, M. E. Roberts, Z. Liu, J. Locklin, Z. Bao, Advanced Materials 20, 2588 (2008).

18. S. C. B. Mannsfeld, M. L. Tang, Z. Bao, Advanced Materials, n/a (2010).

19. Methods and additional results are available as supplementary material on Science Online.

20. J. Rivnay et al., Nat Mater 8, 952 (Dec, 2009).

21. P. Smith, A. J. Pennings, European Polymer Journal 12, 781 (1976).

22. A. J. Lovinger, T. T. Wang, Polymer 20, 725 (1979).

23. R. Z. Rogowski, A. A. Darhuber, Langmuir 26, 11485 (Jul 6, 2010).

24. C. W. Sele et al., Advanced Materials 21, 4926 (2009). 
25. K. H. Kim, D. S. Chung, C. E. Park, D. H. Choi, Journal of Polymer Science Part A: Polymer Chemistry 49, 55 (2011).

26. D. H. Kim et al., Advanced Materials 19, 678 (2007).

27. S. K. Park, T. N. Jackson, J. E. Anthony, D. A. Mourey, Applied Physics Letters 91, 063514 (2007).

28. J. Chen, C. K. Tee, M. Shtein, J. Anthony, D. C. Martin, Journal of Applied Physics 103, 114513 (2008).

29. S. S. Lee et al., Advanced Materials 21, 3605 (2009).

30. $\quad$ Y. Ito et al., Journal of the American Chemical Society 131, 9396 (2009).

31. E. F. Valeev, V. Coropceanu, D. A. da Silva Filho, S. Salman, J.-L.Brédas, J. Am. Chem. Soc., 128, 9882 (2006).

32. A. D. Becke, J. Chem. Phys., 98, 5648 (1993).

33. C. T. Lee, W. T. Yang, R.G. Parr, Phys. Rev. B, 37, 785 (1998).

34. M. M. Francl, W. J. Pietro, W. J. Hehre, W. J, J. S. Binkley, M. S. Gordon, D. J. Defrees, J. A. Pople, J. Chem. Phys., 77, 3654 (1982).

35. W. J. Hehre, R. Ditchfield, J. A. Pople, J. Chem. Phys., 56, 2257 (1972).

36. Y. Shao, L. F. Molnar, Y. Jung, J. Kussmann, C. Ochsenfeld, S. T. Brown, A. T. Gilbert, L. V. Slipchenko, S. V. Levchenko, D. P. O'Neill, R. A. DiStasio, Jr., R. C. Lochan, T. Wang, G. J. Beran, N. A. Besley, J. M. Herbert, C. Y. Lin, T. Van Voorhis, S. H. Chien, A. Sodt, R. P. Steele, V. A Rassolov, P. E. Maslen, P. P. Korambath, R. D. Adamson, B. Austin, J. Baker, E. F. Byrd, H. Dachsel, R. J. Doerksen, A. Dreuw, B. D. Dunietz, A. D. Dutoi, T. R. Furlani, S. R. Gwaltney, A. Heyden, S. Hirata, C. P. Hsu, G. Kedziora, R. Z. Khalliulin, P. Klunzinger, A. M. Lee, M. S. Lee, W. Liang, I. Lotan, N. Nair, B. Peters, E. Proynov, P. A. Pieniazek, Y. M. Rhee, J. Ritchie, E. Rosta, C. D. Sherrill, A. C. Simmonett, J. E. Subotnik, H. L. Woodcock, W. Zhang, A. T. Bell, A. K. Chakraborty, D. M. Chipman, F. J. Keil, A. Warshel, W. J. Hehre, H. F. Schaefer, J. Kong, A. I. Krylov, P. M. Gill, M. Head-Gordon, M. Phys Chem Chem Phys, 8, 3172 (2006).

37. J. E. Norton, J. L. Brédas, J. Chem. Phys., 128, 034701 (2008).

38. K. Senthilkumar, F. C. Grozema, F. M. Bickelhaupt, L. D. A Siebbeles, J. Chem. Phys., 119, 9809 (2003). 